\title{
10. Sterben im Krankenhaus
}

\author{
André-Sebastian Zank-Wins
}

Das Krankenhaus ist ein ungünstiger Ort für sterbende Menschen. Krankenhausbehandlungen intendieren Genesung oder zumindest Stabilisierung der gesundheitlichen Situation. Sollten therapeutische Maßnahmen mit dieser Zielsetzung medizinisch nicht mehr indiziert oder vom Patienten (z.B. im Rahmen einer Patientenverfügung) nicht mehr gewünscht sein, so bemüht man sich schnellstmöglich um eine Verlegung zurück in die Häuslichkeit, in ein Pflegeheim oder gar ein Hospiz.

Dennoch wird im Krankenhaus gestorben! Die Verlegung eines sterbenskranken Menschen ist häufig nicht mehr möglich. Nun gilt es, die bestmöglichen Rahmenbedingungen für den Sterbenden zu realisieren. Ein gutes Zusammenspiel aller vorhandenen Berufsgruppen ist hierbei von erheblicher Bedeutung.

Was aber benötigt ein sterbenskranker Mensch? Dame Cicely Saunders, die Hauptinitiatorin der neuzeitlichen Hospizbewegung, hat vom „total pain“ gesprochen, dem „totalen Schmerzerleben“ eines sterbenskranken Menschen, dem es auf fünf verschiedenen Ebenen zu begegnen gilt:

Total pain (Saunders 2001)

- physisch

- psychisch

- sozial

- spirituell 
- „staffpain“ (Mitarbeiterschmerz)

\section{Der physische Schmerz}

Hauptbedürfnis eines sterbenskranken Menschen ist die wirksame Linderung aller ihn beeinträchtigenden körperlichen Symptome. Schmerzen, Atemnot, Übelkeit, Obstipation, Angst, Schwäche, etc. können unerträgliche Qualen bewirken. Der Betroffene ist auf fachlich kompetente und äußerst schnelle Hilfe angewiesen.

- Informieren Sie sich, wo es in Ihrem Haus palliative Kompetenz oder vielleicht sogar einen palliativmedizinischen Konsiliardienst gibt!

- Handeln Sie schnell!

\section{Der psychische Schmerz}

Die Emotionen von sterbenskranken Menschen können ein ganzes Spektrum zwischen Aggression und Demoralisation (Depression) umfassen und individuell verschieden ausgeprägt sein. Die Angst vor dem Sterben und nahenden Tod kann schockartige bis psychotische Reaktionen hervorrufen, auch (wach-)traumartige Phantasiebildungen können entstehen. Sterbephasenmodelle, wie z.B. das von Elisabeth Kübler-Ross, sind für das Verstehen hilfreich, wenn sie als Interpretationshilfe und nicht schematisch genutzt werden! Alle solche psychischen Phänomene sind prinzipiell erst einmal als seelisch völlig normale(!) Reaktionen am Ende eines Lebens zu bewerten. Sie bedürfen in der Regel mehr einer zugewandten mitmenschlichen Begleitung als einer psychotherapeutisch-intervenierenden Behandlung!

- Erlauben Sie dem Sterbenden seine Gefühle!

- Kommen Sie nicht zu schnell auf die Idee, medikamentös zu agieren!

- Gibt es ein Angebot von Seelsorge oder einen Besuchsdienst in ihrem Haus, die Sie einbeziehen können?

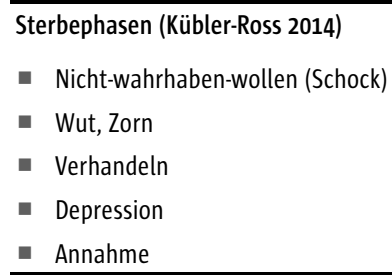

\section{Der soziale Schmerz}

Die Auswirkungen von Krankheit und Sterben auf die sozialen Beziehungsgefüge des Betroffenen können erheblich sein. Der Sterbenskranke findet sich in der Rolle des Bedürftigen vor, manche vertraute Personen ziehen sich zurück, einige wenige werden ihm besonders wichtig. Mit den engsten Bezugspersonen jederzeit in einem geschützten Rahmen zusammen sein zu können, wird zu einem 
der wichtigsten Bedürfnisse. Der Sterbende möchte von seiner Umgebung als „Mensch“ gesehen und angesehen werden, nicht mehr als „Patient“ - d.h. nicht mehr primär in Definition über seine Symptomatik. Mitmensch-sein für den Betroffenen (und seine Angehörigen!), Da-sein, Nähe und Sicherheit vermitteln wird nun zu den zentralen ärztlichen, pflegerischen und interdisziplinären Aufgaben.

- Suchen Sie nach den bestmöglichen Räumlichkeiten für den Sterbenden. Ein angenehm gestaltetes Einzelzimmer mit Rooming-in-Möglichkeit für Angehörige wäre ideal!

- Nehmen Sie sich auf jeder Ihrer Visiten mindestens 5 Minuten Zeit für den Sterbenden! Ziehen Sie Ihren Arztkittel aus und setzen (!) Sie sich zu ihm ans Bett. Bedenken Sie: Sie selbst könnten der Betreffende sein!

\section{Der spirituelle Schmerz}

„Warum muss ich so krank sein?“"Warum muss ich (schon) sterben?“

Die Warum-Frage, die Frage nach Sinn und Unsinn von Krankheit, Leben und Sterben - sie ist die zentral aufbrechende Frage des spirituellen Leidens. Das Hadern mit Gott (religiös) oder dem Schicksal (atheistisch) bedeutet ein Ringen um die Wahrheit der eigenen Existenz, das immer über das irdisch-rationale Verstehen hinausgeht. Medizinische Erklärungen, der Nachweis von innerweltlichen Tun-Ergehen-Zusammenhängen (Ursache-Wirkung) helfen hier nicht weiter, ja führen in die Irre und wirken auf vorhandene Selbstvorwürfe und Schuldgefühle eher verstärkend. Zuhören ist angesagt! Einen Resonanzraum bieten für die inneren Suchbewegungen des Betroffenen. Vertraute (!) religiöse Handlungen können Linderung verschaffen.

- Erfragen Sie religiöse Bedürfnisse. Was lässt sich da ermöglichen?

- Seien Sie vorsichtig mit Impulsen aus Ihrer eigenen religiösen oder spirituellen Tradition. Die Gefahr, dem anderen etwas Fremdes überzuhelfen, ist groß!

- Beziehen Sie die Seelsorge in jedem Fall mit ein! Professionell geschulte Seelsorger haben gelernt, auf das spirituelle Leiden auch von nichtreligiösen Menschen angemessen einzugehen.

\section{Der Mitarbeiterschmerz („staffpain“)}

Stellen Sie sich vor, Sie sind pflege- und hilfebedürftig, aber die Menschen, auf deren Unterstützung Sie angewiesen sind, sind übellaunig, unsensibel und grob. Niemand hat Zeit, niemand hört zu, man „macht an Ihnen herum“, ohne dass Sie sich als Person wahrgenommen fühlen. Das ist „staffpain“! Wer als Arzt oder Pflegekraft „wie am Fließband“ arbeiten muss, läuft Gefahr, dass der Mensch, für den er da (d.h. präsent!) sein sollte, sich auch „wie am Fließband“ behandelt fühlt. Wem z.B. aufgrund eines „Burn-out“ die emotionalen Energien fehlen, sich auf das Gegenüber einzulassen, bewirkt dort ein Gefühl der Beziehungsarmut und Einsamkeit. Die Vermeidung von „staffpain“ steht und fällt zunächst einmal mit einem guten Betriebsklima und einem angemessenen Personalschlüssel. Erst in 
zweiter Linie obliegt es der Selbstsorge des Einzelnen, einen Verlust an Empathie und Beziehungsfähigkeit zu vermeiden.

- Tragen Sie Ihren Teil zu einem guten Betriebsklima und einer guten Kommunikation zwischen allen beteiligten Berufsgruppen bei!

- Versuchen Sie, ein fließbandähnliches Arbeiten zu vermeiden und prüfen Sie, ob es Ihnen gelingt, in jedem Patienten neu den Einzelfall zu sehen!

- Nehmen Sie sich selbst und den Betroffenen ernst. Lassen Sie eigene Ohnmacht zu. Seien Sie ehrlich. Strahlen Sie Ruhe, Verbindlichkeit und Vertrauen aus. Versuchen Sie, in allen Situationen dem anderen gegenüber „ein genießbarer Mensch" zu sein.

- Achten Sie gut auf sich selbst. Bewahren Sie sich Ihre Freude an den Menschen und an Ihrem Beruf. Insbesondere die Sterbenden werden es Ihnen abspüren!

Auch die Angehörigen benötigen Ihre Aufmerksamkeit. Meist sind es nur ein bis wenige Menschen, die für den Sterbenskranken wichtigste Bezugspersonen sind. Sie versuchen, ihm bestmöglich zur Seite zu stehen, haben aber zugleich mit ihren (antizipierten) Trauergefühlen zu kämpfen - Emotionen, die sie insbesondere vor dem Sterbenden häufig zu verbergen suchen. Die Erfahrung von Hilflosigkeit und Ohnmacht kann mit Wut und Aggressionen verbunden sein - auch z.B. gegen die behandelnden Ärzte. Der Wunsch kann stark werden, der Arzt möge doch „retten“ und alles tun, was möglich sei. Angehörige haben eine hohe Schutzbedürftigkeit! Sie wollen verstehen und benötigen dafür klare Informationen. Sie wollen gehört werden und benötigen Menschen, die ihnen zuhören. Sie fühlen sich häufig in einer „Anwaltsrolle“, sodass es für sie besonders wichtig ist, zu erleben, dass mit dem Sterbenden gut umgegangen wird.

- Nehmen Sie sich Zeit für die Angehörigen und beziehen Sie sie aktiv in die Begleitung des Sterbenden ein!

- Bereiten Sie die Angehörigen auf das vor, was kommt - viele haben das Sterben eines Menschen noch nicht miterlebt!

- Organisieren Sie auch hier möglichst eine seelsorgerische Begleitung!

Und wenn der Tod eingetreten ist? Unsere menschliche Würde reicht über den Tod hinaus. Deshalb ist ein ehrfurchtsvoller und sorgsamer Umgang mit dem Verstorbenen von Bedeutung. Auch wenn die Institution Krankenhaus auf ein rasches Entfernen des Leichnams und eine Neubelegung des Platzes drängt, so sollte das ärztliche Handeln sich hier nicht auf die Feststellung des Todes und die Ausstellung des Leichenschauscheins beschränken. Insbesondere die Angehörigen benötigen einen angemessenen Rahmen, sich zu verabschieden. Der letzte Anblick des Verstorbenen prägt sich ihnen unvergesslich ein. Was sie an dieser Stelle erleben, ist für ihren weiteren Trauerweg von allergrößter Relevanz!

- Informieren Sie sich frühzeitig, ob es in Ihrem Krankenhaus ein verabredetes Verfahren oder einen Standard „Umgang mit Sterbenden/Verstorbenen“ gibt. In vielen Häusern wurde darüber schon im Rahmen eines klinischen Ethikkomitees oder eigens dafür gebildeter Arbeitsgruppen beraten. 
- Prüfen Sie, ob der Verstorbene in seinem Zimmer (oder einem eigens dafür eingerichteten Aufbahrungsraum) bleiben kann, bis die Angehörigen sich verabschiedet haben!

- Ermöglichen Sie den Angehörigen in jedem Fall ein Abschiednehmen. (Vorsicht mit Ratschlägen wie: „Behalten Sie ihn lieber in Erinnerung, wie er war.“ Damit verstärken Sie die Angstfantasien der Hinterbliebenen!)

- Entwickeln Sie zusammen mit den Pflegenden eine Kultur des würdevollen Zurechtmachens eines Leichnams (Augen und Mund schließen; ggf. den Verstorbenen waschen; Schläuche, Sonden, Maschinen usw. entfernen; saubere Bettwäsche und Kleidung, ...).

- Treffen Sie Absprachen, wie Sie auch die äußeren Bedingungen im Rahmen einer Abschiedskultur gestalten wollen (z.B. Blumen ins Zimmer, Entzünden eines Gedenklichts [Brandschutz!], u.a.).

- Ermöglichen Sie der Seelsorge in Absprache mit den Angehörigen eine Aussegnung oder auch eine „weltliche“ Verabschiedungsfeier am Bett des Verstorbenen.

\section{Literaturempfehlung}

Borasio GD (2011) Über das Sterben. Was wir wissen. Was wir tun können. Wie wir uns darauf einstellen, C.H. Beck-Verlag München

Kübler-Ross E (2014) Interviews mit Sterbenden. 6. Auflage der erw. Ausgabe von 2009, Kreuz Verlag Freiburg im Breisgau

Saunders C (2001) Hospiz und Begleitung im Schmerz. Herder Verlag Freiburg

Weiß W (1999) Im Sterben nicht allein. Hospiz. Ein Handbuch für Angehörige und Gemeinden. Wichern Verlag Berlin 